\title{
A FREE FLOATING BALL THROMBUS IN THE LEFT ATRIAL CAVITY
}

Costas P. Tsioufis, MD, Christodoulos I. Stefanadis, MD, FACC, Eleftherios G. Tsiamis, MD, Ioannis E. Kallikazaros, MD, and Pavlos K. Toutouzas, MD, FACC, Athens, Greece

A 65-year-old woman with a medical history of mitral valve stenosis was admitted to our hospital with paroxysmal dyspnea, orthopnea, and presyncopal attacks, which started 24 hours before admission. The patient was in atrial fibrillation and had been treated with digitalis, diuretic, $\beta$-blockers, and dicumarole (the last international normalized ratio, 3 days earlier, had been 2.5). Results of physical examination, chest radiography, and electrocardiography were consistent with the underlying mitral valve stenosis. Routine transtho-

From the Department of Cardiology, University of Athens, Hippokration Hospital, Athens, Greece.

Received for publication July 12, 1999; accepted for publication Aug 11, 1999.

Address for reprints: Costas Tsioufis, MD, 4, Athanasiou Diakou St, 15127 Melissia, Greece.

J Thorac Cardiovasc Surg 1999;118:1120-2

Copyright $\odot 1999$ by Mosby, Inc.

$0022-5223 / 99 \$ 8.00+0 \quad \mathbf{1 2 / 5 4} / \mathbf{1 0 2 0 8 2}$ racic echocardiography revealed a stenotic mitral valve and a large left atrium with an occasional appearance of a floating mass within the atrium but not well visible. Transesophageal evaluation soon after revealed a large, round ball-like thrombus with well-demarcated borders, which was free floating in the left atrial cavity, occluding the stenotic mitral valve transiently but completely. The stenotic mitral valve seemed to protect the patient from systemic embolization by the thrombus. The variation in position was not coincident with the cardiac cycle, and the motion of the thrombus was unpredictable (Fig 1).

The patient subsequently underwent urgent cardiac operation with removal of the circular mass from the left atrium and mitral valve replacement. Indeed, no attachment of the mass was found at operation, and the histologic examination proved that it was a thrombus. She was discharged on postoperative day 7 in stable condition.

Discussion. Left atrial ball thrombus appears to be an unusual occurrence. Wood, who first applied the term ball thrombus to this entity in 1814, described autopsy findings in 

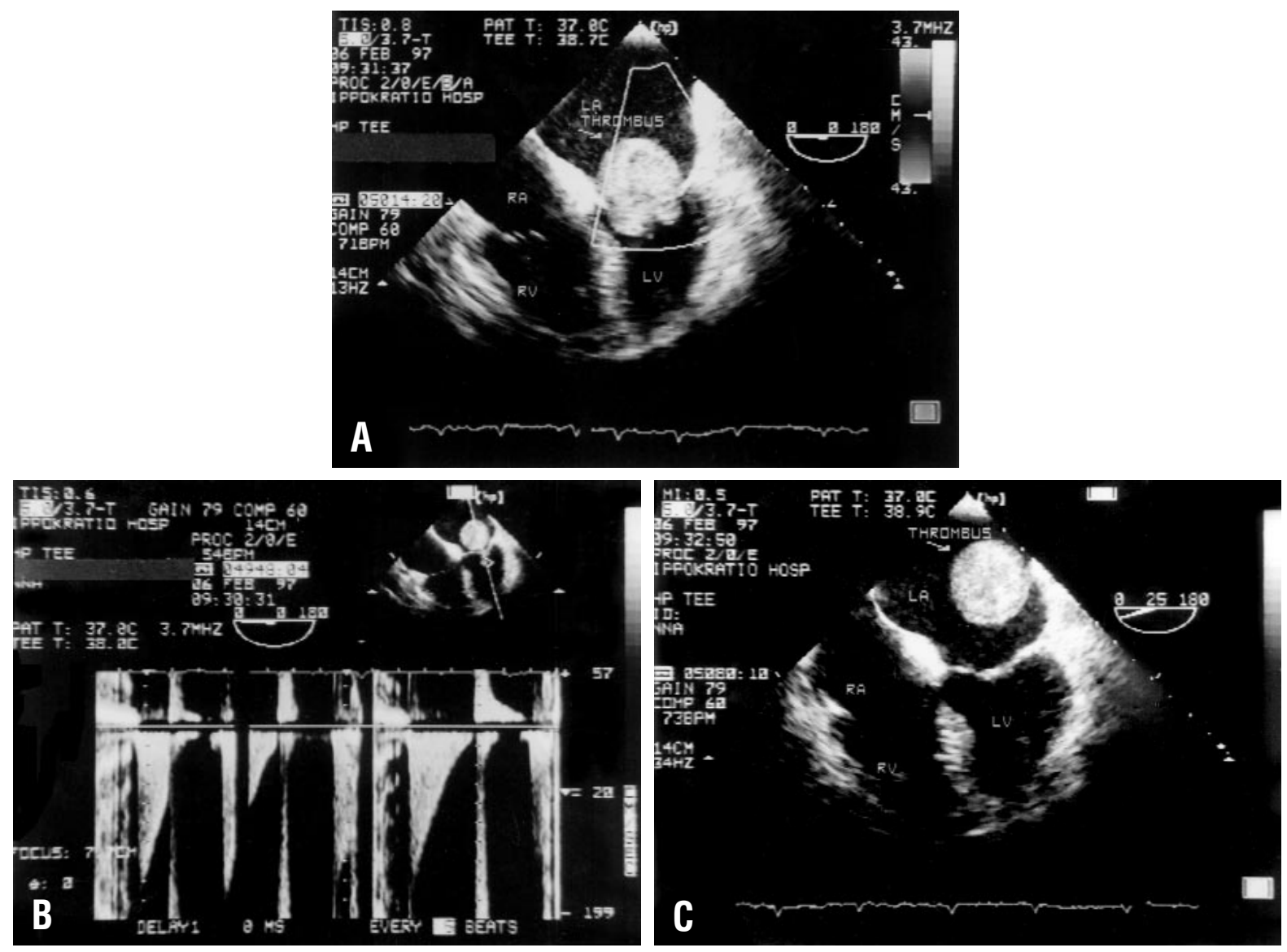

Fig 1. A transesophageal view shows a true free-floating, ball-like thrombus, moving in unpredictable directions in the left atrium. A, The thrombus is protruding through the mitral leaflet completely occluding the stenotic mitral valve and interrupting the left ventricular filling. The absence of flow through the mitral valve is confirmed by the color Doppler image. $L A$, Left atrium; $L V$, left ventricle; $R A$, right atrium; $R V$, right ventricle. $\mathbf{B}$, The transient and occasional occlusion of mitral valve by the floating thrombus is confirmed by the pulsed Doppler image.

C, The thrombus rebounds free back into the left atrium toward the pulmonary vein orifice.

a 15-year-old girl with rheumatic mitral stenosis and syncope. ${ }^{1}$ Recently the routine use of echocardiography, magnetic resonance imaging, or computed tomography permits antemortem diagnosis; and therapy should thus be directed so that autopsy will no longer be an important means of diagnosis. $^{2-4}$ Mention will be made of the diagnostic distinction between free-floating and pedunculated masses.

The symptoms described in our patient are similar to those reported in earlier literature. ${ }^{1,5}$ In 11 cases published in English since 1976, paroxysmal dyspnea was prominent in 4 cases, syncope and peripheral arterial embolism occurred in 3 cases, and 1 patient died suddenly and unexpectedly. ${ }^{1}$ Also, in the same study, the principal findings on cardiac physical examination were those of mitral stenosis in 10 of the 11 patients as in our case. Transesophageal echocardiography has been recommended as the best choice for identifying the presence of left atrial thrombus and also for guiding further therapy designed to reduce the thromboembolic risk. ${ }^{4,6}$
Leung and colleagues ${ }^{6}$ observed a large series of consecutive patients found to have left atrial thrombus on transesophageal echocardiography; they quantitated their embolic event rate to be about $10.4 \%$ per year and the all cause mortality rate at $15.8 \%$ per year. The presence of free-floating thrombus may be expected to indicate higher embolic potential.

Anticoagulation and thrombolytic therapy do not appear to have a role in the acute management of left atrial ball thrombus, although the importance of anticoagulation in prevention of recurrence is obvious. ${ }^{7}$ Surgical removal of the thrombus with simultaneous treatment of the underlying cause is the first choice treatment with good results in most cases.

\section{REFERENCES}

1. Wrisley D, Giambartolomei A, Brownlee W. Left atrial ball thrombus: review of clinical and echocardiographic manifestations with suggestions for management. Am Heart J 1991:122; 1784-90. 
2. Zur-Binenboim C, Ammar R, Grenadier E, Veisler A, Freud M, Palant A. Detection of round floating left atrial thrombus simulating left atrial myxoma by two dimensional echocardiography. Am Heart J 1985;110:492-3.

3. Pearson A, Labovitz A, Tatineni S, Gomez C. Superiority of transesophageal echocardiography in detecting cardiac source of embolism in patients with cerebral ischemia of uncertain etiology. J Am coll Cardiol 1991;17:66-72.

4. Gottdiener J, Temeck B, Patterson R, Fletcher R. Transient occlusion of the mitral valve orifice by a free floating left atrial ball thrombus: identification by two-dimensional echocardiography. Am J Cardial 1984;53:1730-2.

5. Warda M, Garvin J, Massumkihani H, Hall R. Ascultatory and echocardiographic features of mobile left atrial thrombus. J Am Coll Cardiol 1985;5:379-82.

6. Leung D, Davidson P, Cranney G, Walsh W. Thromboembolic risks of left atrial thrombus detected by transesophageal echocardiogram. Am J Cardiol 1997;79:626-9.

7. Blazer D, Degroat T, Kotler M, et al. Peripheral embolization during thrombolytic therapy for left atrial thrombus. Am J Cardiol 1986;58:554-5. 